\title{
Monitoring Ultrafast Laser Micro-Excitation and Modification Deep inside GaAs
}

\author{
Andong Wang ${ }^{1}$, Amlan Das ${ }^{1}$, Jörg Hermann ${ }^{1}$, David Grojo ${ }^{1}$ \\ 1. Aix-Marseille Université, CNRS, LP3, UMR7341, 13288 Marseille, France
}

Creating 3D micro/nano-structures inside semiconductors, such as Silicon or GaAs, is a key for numerous advanced applications from microelectronics to integrated photonics, micro-electro-mechanical systems (MEMS) manufacturing. Direct ultrafast laser writing introduces a promising alternative to lithographic methods for this purpose because of its capability with focused infrared beams to precisely induce micro/nano-scale structures. This has already been successfully applied in wide bandgap materials, but recent research indicates more severe difficulties for applications in semiconductors [1-3]. The inherent properties of narrow bandgap and large nonlinear refractive index materials lead to strong deteriorations of the ideal laser focusing conditions that prevents to access high space-time energy localization with the shortest pulses [2]. The nonlinear propagation distortions lead to limited fluence delivery and consequently low-quality writing in most cases, or even to the absence of modifications when femtosecond pulses are used. Accordingly, there is a strong motivation to monitor and optimize the conditions inside semiconductors to achieve high-quality 3D fabrication inside semiconductors.

To optimize the process, a first step is to characterize the distortion of the focusing spot. Strong difficulties exist for the measurement because the interaction is under the surface and the dimensions of the laser spot is small, especially in the tight focusing conditions. We propose to image the laser propagation by taking advantage of the photo-emission from the laser-induced free-carriers. Fig. 1a shows the light emission generated by laser focusing inside GaAs materials (similar phenomenon also happens in Silicon). Its shape is similar to the plasma shape measured by ultrafast laser pump-probe shadowgraph (Fig. 1b). By measuring the polarization and spectrum range, the photo-emission is confirmed to be related to radiative recombination. Using a calibration method, we show that its intensity increases linearly with the electron density and thus it can be a useful approach for quantitative analysis of nonlinear ionization inside semiconductors. In addition to 2D images, this method can also be used to reconstruct by a z-scanning 3D distribution of excitations. This offers one more additional dimension for analyses of potentially complex spatial features with non-Gaussian or strongly distorted beams. An example of near-surface plasma induced by looser focusing is shown in Fig. 1c, demonstrating the possibility for 3D imaging.
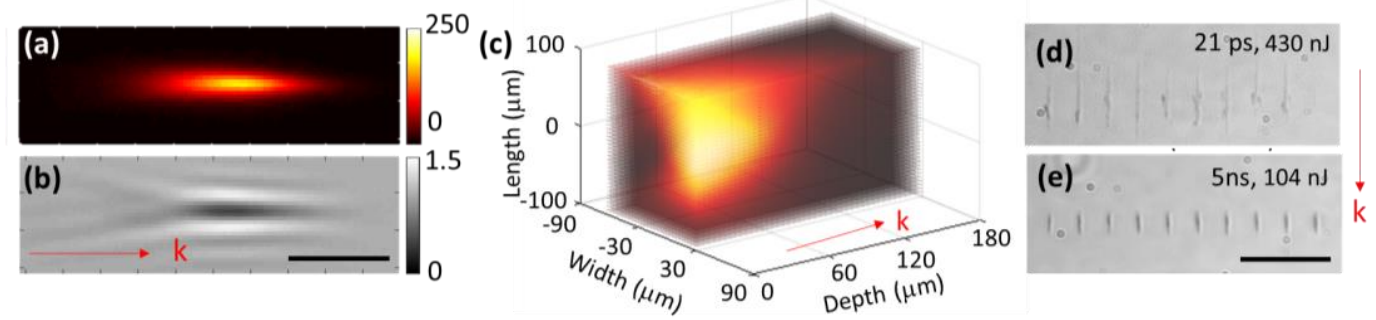

Fig. 1 (a, b) The light emission (a) and the shadowgraph (b) of the plasma focused $300 \mu$ m under the surface of GaAs material. The scale bar is $20 \mu \mathrm{m}$. (c) The 3D reconstruction of light emission from plasma created by NA0.3 lens. Imaging lens is $50 x(\mathrm{NA} 0.65)$. The images only show half of the beam from width -90 to $0 \mu \mathrm{m}$. The depth axis represents the depth below the surface. (d, e) Internal modification inside GaAs by picosecond (d) and nanosecond pulses (e). Scare bar is $30 \mu \mathrm{m}$. The " $\mathrm{k}$ " vector represents the direction of laser beam propagation.

By using the proposed methods, we measured the laser focusing conditions under different pulse durations and various numerical apertures (NA). We quantify the electron density in the pre-focal region that affects the energy delivery for modest focusing conditions and short pulse durations. This allows identifying the most favourable conditions for bulk modifications. Our conclusions are also supported by material modification experiments. Fig. $1(\mathrm{~d}, \mathrm{e})$ shows the modifications produced by picosecond and nanosecond pulses focused with $0.85 \mathrm{NA}$ lens. The results obtained with femtosecond pulses are not shown as severe delocalization prevented from permanent modification. The results in material modification experiments and plasma emission measurements agrees well. They support the conclusion of strong requirements existing for material writing. To process $\mathrm{GaAs}$ or $\mathrm{Si}$ [3], there are systemically more than an energy threshold to exceed in given configuration. Other threshold values applying to the focusing NA and the pulse duration must be inevitably crossed for localized and stable modifications.

Acknowledgement: This work is funded by H2020 European Research Council (724480).

\section{References}

[1] A. Wang, A. Das, D. Grojo. "Ultrafast laser writing inside silicon with THz-repetition-rate trains of pulses". Research, 8149764 (2020). [2] A. Wang, A. Das, and D. Grojo, "Temporal-contrast imperfections as drivers for ultrafast laser modifications in bulk silicon". Physical Review Research, 2, 033023(2020).

[3] A. Das, A. Wang, O. Uteza, D. Grojo, "Pulse-duration dependence of laser-induced modifications inside silicon". Optics Express, 28(18), 26623-26635(2020). 